\title{
Atypical Polypoid Adenomyoma (APAM)
}

\author{
Lubna Javed ${ }^{1}$, Nadia Ashraf ${ }^{1}$, Mashaal Sabqat ${ }^{2}$ and Amtullah Zareen $^{1}$ \\ ${ }^{1}$ Department of Gynae Unit 1, Jinnah Hospital, Lahore, Pakistan \\ ${ }^{2}$ Department of Medical Education, Foundation University, Islamabad, Pakistan
}

\begin{abstract}
Atypical polypoid adenomyoma (APAM) is a rare polypoidal benign tumor of uterus, which usually presents with irregular vaginal bleeding in women of reproductive age. It has the potential of malignant transformation but does not metastasise. It may coexist with endometrial hyperplasia and adenocarcinoma; and so, is usually misdiagnosed. It is composed of atypical endometrial glands along with bundles of smooth muscle fibres. It has a high incidence of recurrence. We describe a case of APAM, which was managed at a tertiary care hospital. A 25-year woman with irregular vaginal bleeding and abdominal mass had total abdominal hysterectomy (TAH) on suspicion of endometrial sarcoma. Histopathology specimen revealed APAM. On follow-up so far, she is in stable condition and asymptomatic.
\end{abstract}

Key Words: Atypical polypoid adenomyosis, Adenosarcoma, Endometrial carcinoma.

How to cite this article: Javed L, Ashraf N, Sabqat M, Zareen A. Atypical Polypoid Adenomyoma (APAM). J Coll Physicians Surg Pak 2021; 31(06):719-721.

\section{INTRODUCTION}

Atypical polypoid adenomyoma (APAM) is a rare benign polypoidal tumor of uterus, reported first by Mazur in $1981 .{ }^{1}$ It has the potential of malignant transformation and a high incidence of recurrence, but does not metastasise. Endometrial carcinoma coexists with APAM in $8.8 \%$ of cases. ${ }^{2}$ APAM is composed of atypical endometrial glands surrounded by bundles of smooth muscle fibres and fibroblasts. ${ }^{3}$ It has an appearance similar to endometrial carcinoma, adenosarcoma and carcinosarcoma, so its diagnosis is usually missed. The age range for its occurrence is $21-73$ years with a mean of 38 years. ${ }^{4}$ So far, 237 cases have been reported worldwide.

Patients usually present with abdominal mass and irregular vaginal bleeding. There is strong association with infertility. The uterus is enlarged with endometrial cavity containing multiple polypoidal growths raising suspicion of adenosarcoma or endometrial carcinoma.

Ultrasound appearance is of a lobulated mass with cystic and hemorrhagic foci, vascular pedicle and associated adenomyosis. Low intensity areas are observed in T2-weighted magnetic resonance imaging (MRI). MRI and ultrasound are helpful in diagnosis but confirmation is by histopathological examination of the lesion.

Correspondence to: Dr. Lubna Javed, Department of Gynae Unit 1, Jinnah Hospital, Lahore, Pakistan

E-mail: lubna_javed@live.com

Received: January 14, 2020; Revised: March 02, 2020;

Accepted: March 11, 2020

DOI: https://doi.org/10.29271/jcpsp.2021.06.719
Management is individualised according to age, clinical features and fertility desires. Fertility sparing modes of management include endometrial ablation and long-term use of high dose progestogens, but requires long-term follow-up because of its high risk of recurrence. We present here a case of a young woman with APAM, which was managed at our hospital.

\section{CASE REPORT}

A 25-year nulliparous woman married for 7 years, presented with 5 years history of heavy vaginal bleeding. She also had history of post-coital bleeding and dyspareunia. For her symptoms, she had been having different treatments with no relief. Later, she was found to have a polypoidal growth projecting out of cervix, for which she underwent polypectomy three years back at a private clinic. The histopathology report of polypoidal growth turned out to be endometrial hyperplasia. She was given different hormone preparations, but her symptoms did not improve. She also took multiple cycles of ovulation induction drugs but was unable to conceive. She gradually developed worsening symptoms including irregular heavier periods, weight loss and generalised weakness, for which she presented to Jinnah Hospital, Lahore. On examination, she was pale looking. There was a 20 weeks size firm mass in lower abdomen arising out of pelvis, and lower limit of mass was not reachable. On vaginal examination, there was $8 \times 6 \mathrm{~cm}$ rounded mass occupying upper two-thirds of vagina with a broad stalk extending into uterine cavity; cervix could not be visualised. Ultrasound scan was done which showed bulky uterus with $8.5 \times 5.8 \mathrm{~cm}$ size echogenic mass in the endometrial cavity and endo-cervical canal. Moreover, multiple small cystic areas were seen within the mass. MRI was also carried out, which reported uterus measuring $17.4 \times 8.6 \times 12.4 \mathrm{~cm}$, with diffusely thickened and heterogeneous endometrium measuring $4.9 \mathrm{~cm}$ containing 
innumerable cystic areas completely occupying endometrial cavity (Figure 1). Extension was noted in the cervical canal with cervical stroma involvement and myometrial invasion. Returning signals were hypo-intense on T1-weighted imaging (T1WI) and hyper-intense on T2WI, suggesting stage ll endometrial carcinoma. Her examination under anesthesia (EUA) and biopsy was planned, which was carried out. On EUA, a $6 \times 8 \mathrm{~cm}$ polypoidal growth was seen obscuring approach to the endometrial cavity. A $2 \times 2 \mathrm{~cm}$ tissue biopsy was taken from the polypoidal mass as the uterine cavity could not be explored. Histopathology report showed endometrial polyp.

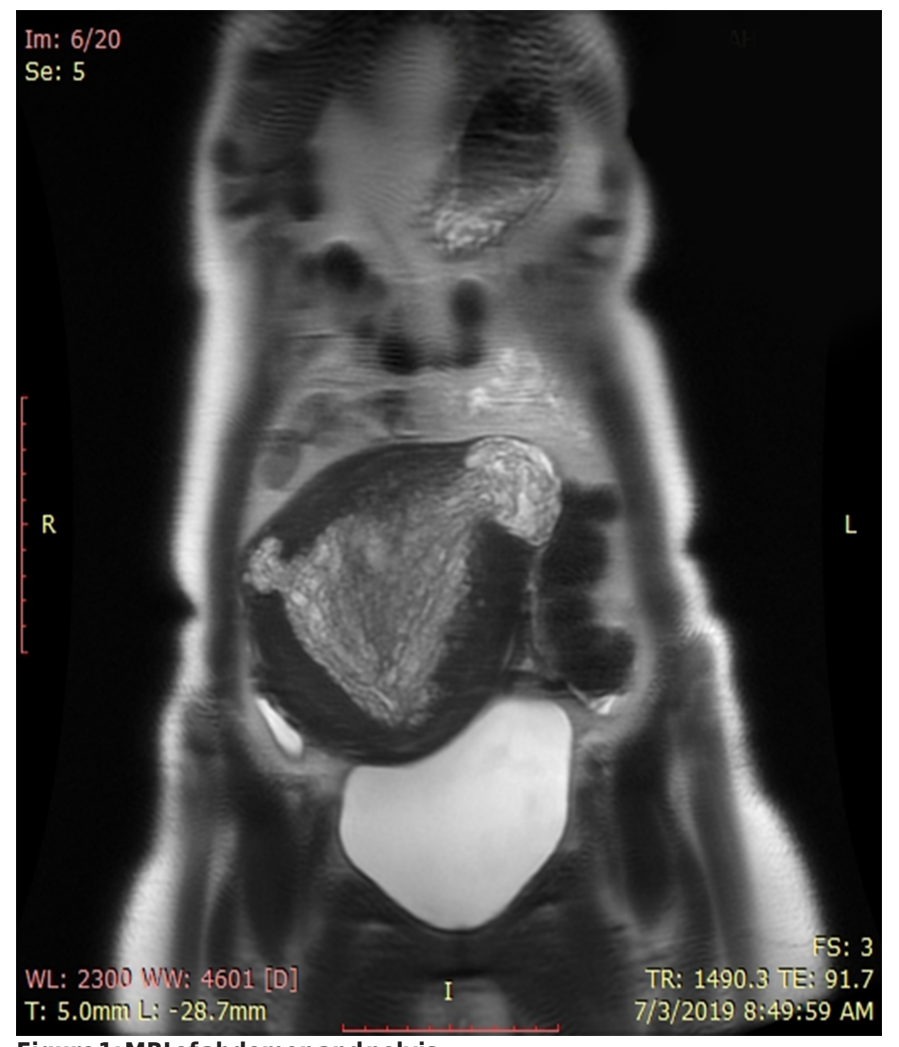

Figure 1: MRI of abdomen and pelvis.

Keeping in view her symptoms, clinical examinations, ultrasound and MRI findings, laparotomy was planned. Patient was counselled regarding the possibility of hysterectomy. Preoperatively, she was transfused two units of blood with four units in hand for surgery. Her surgery was performed. Vaginal growth was removed vaginally, and uterus was seen to be full of multiple polypoidal masses, so laparotomy was proceeded with. Sub-umbilical midline incision was given and peritoneal washings were taken. Intraoperatively, uterus was found to be irregularly enlarged to 20 weeks size. An incision was given in the anterior lower uterine wall and the cavity was approached with the idea to remove the growth and leave uterus behind. Numerous small polypoidal growths were found to be filling the whole cavity with thick uterine walls raising the suspicion of adenosarcoma or adenocarcinoma (Figure 2). As there was a strong suspicion of malignancy, total abdominal hysterectomy was proceeded with. Both tubes and ovaries were normal looking and were preserved. Rest of the abdominal and pelvic organswereunremarkable. Patienthad an uneventful postoper- ative recovery and was discharged on $4^{\text {th }}$ postoperative day. Her histopathology report was of APAM with no evidence of invasion or malignancy (Figure 3).

Fluid cytology was negative for malignant cells. Stitches were removed on $10^{\text {th }}$ postoperative day. On follow-up so far, she is in stable condition and asymptomatic.

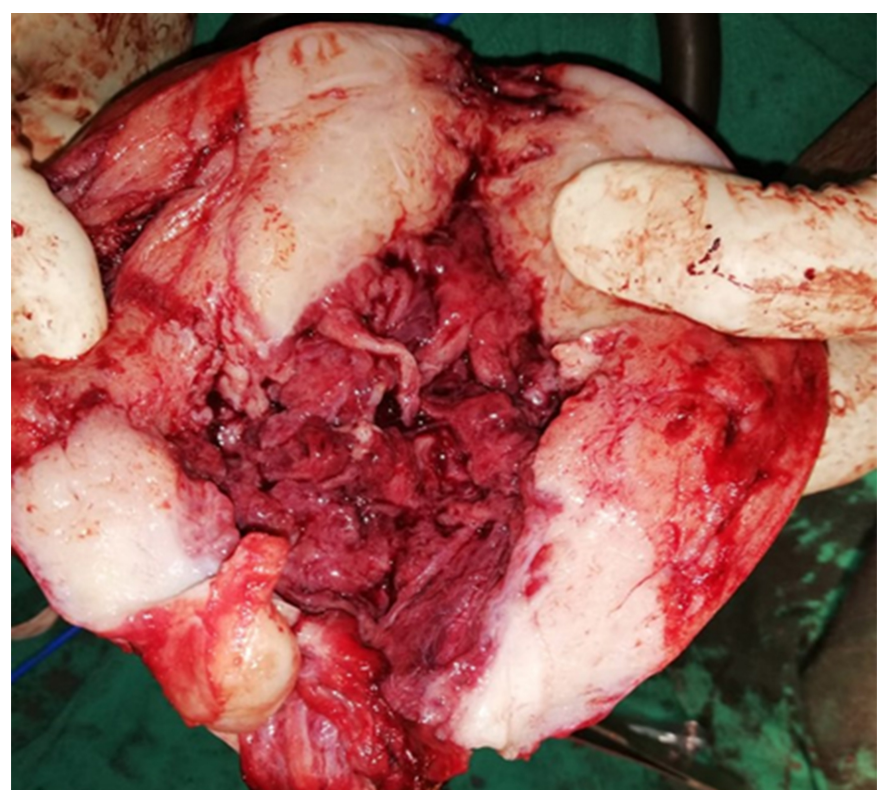

Figure 2: Gross appearance on cut section.

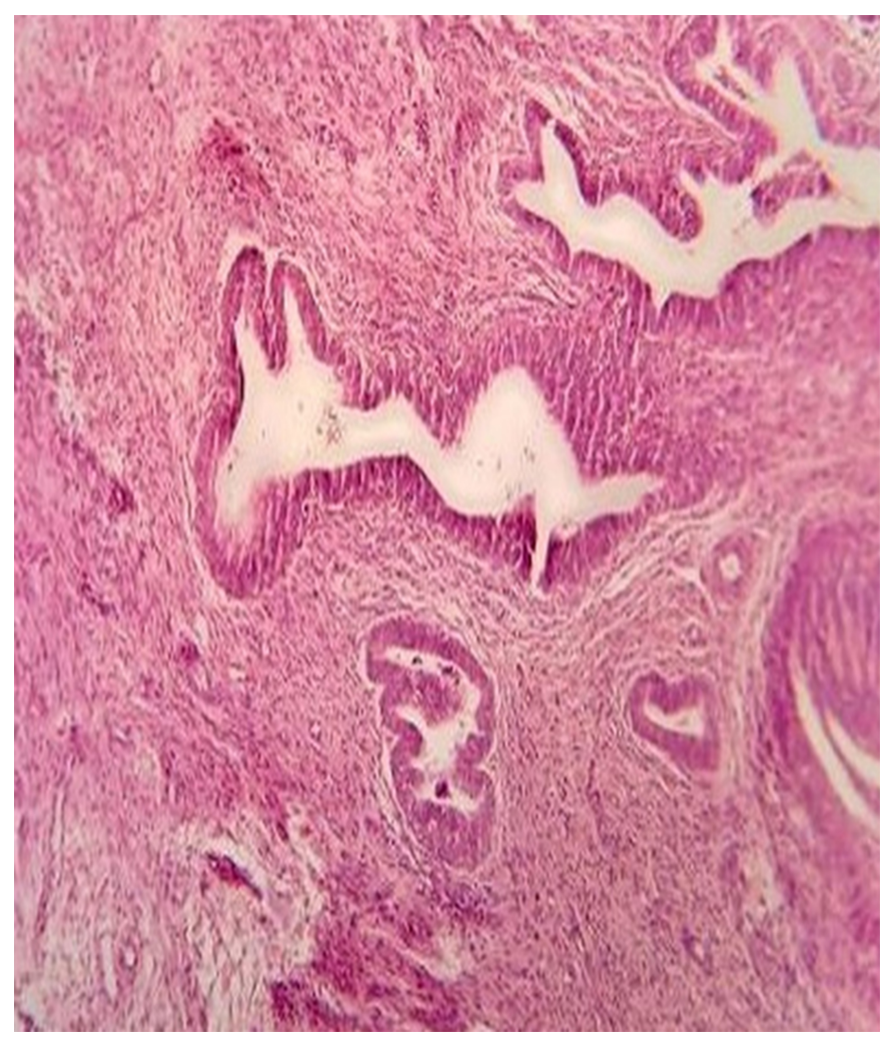

Figure 3: Histopathological appearance $(H \& E, \times 40)$.

\section{DISCUSSION}

APAM is an uncommon benign uterine lesion, which is often misdiagnosed because of its association with atypical hyperplasia or 
adenocarcinoma. It has mixed epithelial and mesenchymal components, is non-invasive, does not metastasise, but recurs after uterus-sparing management. ${ }^{5}$ Advanced age and prolonged estrogen stimulation are considered as risk factors. It has a strong association with infertility. ${ }^{6}$ Clement et al. described three cases of APAM in women with Turner's syndrome. These patients were on long-term estrogen use and presented with irregular vaginal bleeding and had cervical polyps. Histopathology of polyps showed APAM, suggesting that APAM may be a complication of prolonged estrogen stimulation. ${ }^{7}$ Sajjad et al. reported a case of a patient who presented with urinary retention. The patient had a large heterogeneous growth with cystic spaces in upper third of vagina, which turned out to be APAM on histopathology. ${ }^{8}$ This unusual presentation may be due to pressure effect of uterine mass. Ultrasound and MRI are suggestive but not conclusive for the diagnosis of APAM. MRI shows polypoidal mass with mixture of hypo-intensity and hyper-intensity on T2-weighted MR images and contrast study reveals irregular enhancement of tumor. ${ }^{9}$ Similarly, imaging in our patient showed enlarged uterus filled with polypoidal growths with cystic areas and enhancement of contrast on T2WI. As APAM is not a common condition and its appearance overlaps with adenocarcinoma and endometrial sarcoma, hence the radiologist suggested possibility of stage II endometrial carcinoma in our case. The management of APAM depends upon age, symptoms and fertility desires of the patient.

There are fertility sparing modes of treatment like prolonged use of high dose progestogens and trans-cervical resection of growth with removal of sufficient muscle layer of uterus resulting in favourable fertility outcome. ${ }^{10}$ In these cases, long-term follow-up with hysteroscopic biopsy is required because of a high risk of recurrence and malignant transformation. ${ }^{7}$

Hysterectomy is the treatment of choice in older patients or patients with recurrent disease. ${ }^{3}$ Our patient although was nulliparous and young but she had recurrent disease which was missed previously, was associated with hyperplasia and had a strong suspicion of carcinoma on imaging and gross appearance, and since it was not possible to remove innumerable polypoidal growths. Therefore, we proceeded with hysterectomy as a definite management.

APAM is a rare condition and experience with its diagnosis and optimal management is limited. It should be differentiated from endometrial carcinoma and sarcoma and whether it is pre-cancerous for endometrial carcinoma is unclear yet. Management should beindividualized.

\section{CONFLICT OF INTEREST:}

Authors declared no conflict of interest.

\section{PATIENT'S CONSENT:}

The consent of the patient was taken prior to the writing of the manuscript.

\section{AUTHORS' CONTRIBUTION:}

LJ: Selection of the case, conception of the idea, layout of the manuscript, collection of information and ensuring its accuracy and integrity.

NA: Drafting of the manuscript.

MS: Critical revision and final editing.

AZ: Overall supervision and final approval of the version to be published.

\section{REFERENCES}

1. Mazur MT. Atypical polypoid adenomyomas of the endometrium. Am J Surg Pathol 1981; 5(5):473-82. doi: 10.1097/00000478-198107000-00006.

2. Zhang HK, Chen WD. Atypical polypoid adenomyomas progressed to endometrial endometrioid adenocarcinomas. Arch Gynecol Obstet 2012; 286(3):707-10. doi: 10.1007/s00404-012-2355-x.

3. Bo Ma, Zhu Y, Liu Y. Management of atypical polypoid adenomyoma of the uterus, a single-centre study. Medicine (Baltimore) 2018; 97(12): doi: 10.1097/MD. 00000000000 10135.

4. Matsumoto T, Hiura M, Baba T, Ishiko O, Shiozawa T, Yaegashi $N$, et al. Clinical management of atypical polypoid adenomyoma of uterus. A clinicopathological review of 29 cases. Gynecol Oncol 2013; 129(1):54-7. doi: 10.1016/j.ygyno.2012.12.040.

5. Němejcová K, Kenny SL, Laco J, Petr S, Staněk L, Zikán M, et al. Atypical polypoid adenomyoma of the uterus: An immunohistochemical and molecular study of 21 cases. Am J Surg Pathol 2015; 39(8):p 1148-55. doi: 10.1097/ PAS. 0000000000000428.

6. Kssu L, Banno K, Yanokura M, Kobayashi Y, Ueki A, Ono A, et al. J Cancer Therapy 2011; 2:458-62. Doi: 10.4236/ jct.2011.24061.

7. Clement PB, Young RH. Atypical polypoid adenomyoma of the uterus associated with Turner's syndrome. A report of three cases, including a review of "estrogen-associated" endometrial neoplasms and neoplasms associated with Turner's syndrome. Int J Gynecol Pathol 1987; 6(2):104-13. doi: 10.1097/00004347-198706000-00002.

8. Sajjad N, Iqbal H, Khandwala K, Afzal S. Polypoid adenomyoma of uterus. Cureua 2019; 11(2):e4044. doi:10.7759. doi: 10.7759/cureus.4044.

9. Maeda T, Tateishi U, Sasajima Y, Hasegawa T, Daisaki H, Arai $Y$, et al. Atypical polypoid adenomyoma of uterus: Appearance on F-FDG PET/MRI Fused Images. Am J Roentgenol 2006; 186(2):320-3. Doi:10.2214/AJR.04.1799.

10. Viols GA, Ettler HC. Atypical polypoid adenomyoma and hysteroscopic endometrial ablation. J Obstetrics Gynecol Canada 2003; 25(9):760-2. doi: 10.1016/s1701- 2163(16) 31005-2. 